\title{
IgA levels in lactating dairy goats fed diets including greenhouse wastes
}

\author{
M. Romero-Huelva, L. Abecia, D. R. Yáñez-Ruiz and E. Molina-Alcaide \\ Estación Experimental del Zaidín (CSIC), Professor Albareda, 1, Granada, Spain
}

Some dietary manipulations in ruminants feeding can result in methane reduction ${ }^{(1,2)}$. Antimethanogenic mechanisms involve changes in the rumen microbiota which may be associated with modifications in the host immune system. The aim of this work was to study the effect of the partial substitution of concentrate in lactating goat's diet with multinutrient feed blocks (FB) including unconventional feedstuffs such as greenhouse horticulture wastes (fruits of tomato and cucumber).

Eight Murciano-Granadina goats in the middle of the third lactation were used to carry out four 39-d trials following a $4 \times 4$ Latin square experimental design, to study four experimental diets: alfalfa hay plus concentrate in a $1: 1$ ratio (diet AC) or 1:0.65 and feed blocks including wastes of tomato fruits (diet ACT), cucumber (diet ACC) or barley (diet ACB). In each trial 2 goats were randomly assigned to one of the dietary treatments. The average intakes of feed blocks including tomato, cucumber and barley were 231 (SE 68.7), 238 (SE 58.9) and 223 (SE 88.4) g of fresh matter per animal and day, respectively. However, total DM intake $(P=0.085)$ and body weight $(P=0.411)$ were similar (Table). From day 36 to 39 animals were moved to a set of four open circuit respiration chambers for methane emissions measurement. To assess the effect on immune system of the animal, total IgA concentration was measured in plasma by ELISA.

The replacement of $35 \%$ of concentrate with feed blocks including tomato or cucumber wastes did not have any effect $(P=0.544)$ on total IgA antibody levels in plasma. Compared to the control diet, methane emissions (Figure) were $39 \%$ lowered $(P<0.001)$ in diets including tomato and cucumber FB and $29 \%$ in animals receiving ACB diet, which is environmentally relevant.

Table 1. Body weight $(\mathrm{kg})$, total DM intake $(\mathrm{g})$ and total $\mathrm{IgA}(\mathrm{ng} / \mathrm{mL})$ antibody levels in plasma of lactating dairy goats fed the experimental diets $(n=8)$

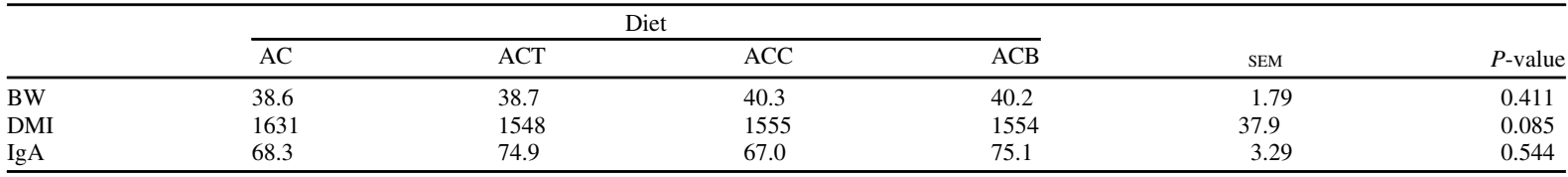

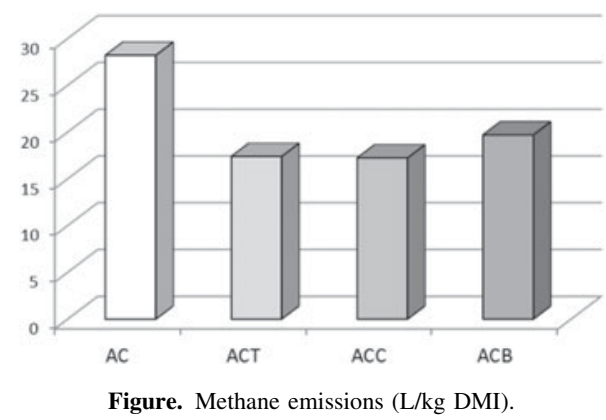

Our study suggests that the inclusion of greenhouse wastes in lactating goat's diet did not change the IgA levels in plasma although diets including greenhouse wastes showed anantimethanogenic potential.

1. Ben Salem H (2010) RevBrasZootecn 39, 337-347.

2. Martín C, Morgavi D \& Doreau M. (2010) Animal 4, 351-365. 